\title{
A Kinetic Study of the Colony Growth of Streptomyces coelicolor A3(2) and J802 on Solid Medium
}

\author{
By EUNICE J. ALLAN† AND J. I. PROSSER* \\ Department of Microbiology, University of Aberdeen, Marischal College, \\ Aberdeen $A B 9$ IAS, UK
}

(Received 3 December 1984; revised 25 April 1985)

\begin{abstract}
Colony growth kinetics of Streptomyces coelicolor A3(2), the wild-type strain, and J802, a mutant incapable of utilizing agar as the sole carbon and energy source, have been studied on solid medium. General features comparable to colony growth of filamentous fungi were observed in both streptomycete strains. Hyphal frequency at the margins of colonies was assessed as the number of hyphae crossing an arc of defined length and increased in an exponential manner with distance from the margin, reaching a maximum at approximately $200 \mu \mathrm{m}$. Aerial hyphal initials were formed approximately $350 \mu \mathrm{m}$ from the margin. Colony radial extension was linear, but in the wild-type strain grown at low medium depths, growth was sometimes multiphasic, with successive phases of linear growth each exhibiting a slower radial growth rate than that of the preceding phase. Hyphal frequency at the colony margin decreased as colony diameter increased, indicating significant changes in environmental conditions in the peripheral growth zone during colony growth. In the primary phase of linear growth, the colony radial growth rate of both strains was independent of medium depth and in the absence of a cellophane membrane was independent of glucose concentration. Colony radial growth rate of strain J802 growing on a cellophane underlay increased with glucose concentration. Hyphal frequency of strain $A 3(2)$ increased gradually with medium depth but was independent of glucose concentration. In strain $\mathrm{J} 802$, hyphal frequency exhibited a strong dependence on both medium depth and glucose concentration. A threshold glucose concentration for growth was not found and inhibition of strain A3(2) occurred above $1 \mathrm{~g} \mathrm{1}^{-1}$. The results agree with many aspects of accepted theories for colony growth of both unicells and filamentous fungi, but suggest production of staling products and/or secondary metabolites to be of equal significance to nutrient limitation in controlling colony development.
\end{abstract}

\section{INTRODUCTION}

A model describing the surface growth of microbial colonies was first proposed by Pirt (1967). This model predicts an exponential increase in colony diameter until nutrients become depleted at the colony centre. Subsequent radial extension is linear and results from exponential growth within a peripheral zone of constant width. Linear radial expansion has been observed experimentally in bacterial colonies (Pirt, 1967; Palumbo et al., 1971; Cooper et al., 1968; Reyrolle \& Letellier, 1979) but it subsequently declines so that discrete colonies are generally formed and medium is not fully colonized. This is thought to result from the restriction to nutrient supply as the region of nutrient depletion reaches the base of the agar layer (Cooper $\mathrm{et}$ al., 1968). The duration of the linear growth period is therefore proportional to agar depth (Pirt, 1975). In addition, the colony radial growth rate increases with medium depth, up to a maximum value, and is proportional to the square root of the initial substrate concentration. The final size

† Present address: Wolfson Institute of Biotechnology, University of Sheffield, Sheffield S10 2TN, UK.

Abbreviation: MM, minimal medium. 
achieved by bacterial colonies is hence dependent on the above factors in addition to the characteristics of the organism itself; for example, morphology and motility affect oxygen diffusion into the colony (Wimpenny \& Lewis, 1977).

Colony growth of filamentous fungi is similar to that of bacteria in that radial extension is initially exponential and subsequently linear with exponential growth occurring in a peripheral growth zone of constant width (Trinci, 1971). However, their colony radial growth rate is typically independent of medium depth while the effect of glucose concentration is less well defined and varies between species (Trinci, 1969). This results from the ability of filamentous fungi to control the rate of branch initiation, in response to variation in environmental conditions, permitting maintenance of maximum rates of hyphal extension, and therefore colony radial growth rate, while optimizing utilization of substrate behind the colony margin. For example, branching at the margin of colonies of Aspergillus nidulans increases with medium depth although colony radial growth rate is unaffected, while increased glucose concentration results in increases in both branching and rate of colony expansion (Trinci, 1969). Similar effects have also been reported for Geotrichum candidum by Robinson \& Smith (1979). Under improving nutrient conditions, therefore, more branches are initiated, thus increasing surface colonization and maximizing substrate utilization. As a corollary, any change in hyphal frequency will reflect environmental changes such as nutrient status of the medium or production of inhibitors from within the colony (e.g. secondary metabolites) and antagonistic effects from neighbouring colonies. The filamentous growth form also permits spatial and temporal differentiation of both morphology and genetic composition within a single organism (Bonner, 1973). Unicellular organisms are generally not capable of such control and typically form discrete colonies whose size is limited by substrate diffusion into a peripheral growth zone of high biomass density. In addition, a threshold glucose concentration appears necessary for bacterial colony growth (Pirt, 1967) but not for fungi, which are capable of growing at extremely low glucose concentrations (Trinci, 1969).

Hyphal density has previously been defined as either the number of branches which cross an arc of specific length (Trinci, 1969; Robinson \& Smith, 1979) or the length of hyphae per unit area (Plomley, 1959); in Aspergillus nidulans and Chaetomium globosum hyphal density increases exponentially with distance from the colony margin, until a maximum, saturation value is attained. We have adopted the former method in this study but have used the term hyphal frequency rather than hyphal density. The hyphal growth unit, defined as the total mycelial length/total number of hyphal tips, is also a measure of the extent of branching and achieves a constant value for the entire mycelium of young colonies of both fungi (Trinci, 1974) and actinomycetes (Allan \& Prosser, 1983). It is generally assumed that a constant value is maintained at the margin throughout colony growth.

Actinomycetes typically form colonies similar in size to those of unicellular bacteria but, despite lower hyphal extension rates and diameters, the initial mycelial growth kinetics of the filamentous actinomycete Streptomyces coelicolor are similar to those of filamentous fungi (Allan \& Prosser, 1983). Visual observations indicate that the mycelial mat is sparse under low nutrient conditions and Riesenberg \& Bergter (1979) found an increase in branching frequency at high specific growth rates in glucose limited continuous cultures of Streptomyces hygroscopicus. They suggested that apical extension rate could not increase at the higher growth rates and that biosynthesis was therefore directed towards branch formation. In addition, secondary metabolites and staling compounds have been implicated in changes occurring during development of colonies of filamentous fungi (Park \& Robinson, 1966; K. M. McLean \& J. I. Prosser, unpublished results) and streptomycete colony development may be similarly affected. The current investigation was therefore undertaken to determine the significance of such factors in actinomycete growth and branching and to test the applicability of the model of Pirt (1967).

\section{METHODS}

Organisms. Two strains of Streptomyces coelicolor were used. The wild-type, strain A3(2), which is capable of degrading agar and utilizing the products for growth, and a mutant, strain J802, which produces a diffusible agarase but is unable to utilize agar as a carbon and energy source (Hodgson \& Chater, 1981). The taxonomy of 
strain A3(2) is confused but its characteristics closely resemble the type species of Streptomyces violaceoruber (Shirling \& Gottlieb, 1968). This identification has been confirmed by a computer-assisted identification system (E.M.H. Wellington, personal communication). However, as the name $S$. coelicolor is commonly used in research involving this organism (Pridham \& Tresner, 1974) it will be used throughout this text. Both strains were kindly supplied by Professor D. A. Hopwood and Dr K. F. Chater from the culture collection of the John Innes Institute, Colney Lane, Norwich, UK. The non-agar-utilizing phenotype of strain J802 was continually checked for reversion by its sparse growth on water agar (Purified Agar, Oxoid) and its delayed production of agarase compared to the wild-type. Agar degradation was monitored by staining plates with Gram's iodine $(0 \cdot 026 \mathrm{M}$-iodine in $0.04 \mathrm{M}$-potassium iodide) resulting in a pale yellow/brown region in the area of polysaccharide breakdown against a dark brown background. In colonies grown from single spores, the wild-type produced agarase within $2 \mathrm{~d}$ of culture at $30^{\circ} \mathrm{C}$ compared to $7 \mathrm{~d}$ for strain $\mathrm{J} 802$.

Media. Spores of both strains were obtained from frozen stocks originally harvested from oatmeal agar and maintained in $10^{\circ}(\mathrm{v} / \mathrm{v})$ glycerol by the method of Allan \& Prosser (1983). To investigate the minimum glucose concentration required for growth, dilute spore suspensions (approximately $10^{3}$ spores $\mathrm{ml}^{-1}$ ) were prepared without using the detergent Tween 80, which may act as a growth substrate (Bradley, 1978). Organisms were routinely cultured on minimal medium (MM; Hopwood, 1960) containing Oxoid Agar No. $3(1.5 \%$, w/v) and, unless otherwise stated, at a final glucose concentration of $0.4 \mathrm{~g} \mathrm{l}^{-1}$ poured to a depth of $3.25 \mathrm{~mm}$. The glucose requirement for growth and sporulation of both strains was investigated by subculturing on $\mathrm{MM}$ with glucose concentrations of $0,0 \cdot 001,0 \cdot 01$ and $0.4 \mathrm{~g} \mathrm{l}^{-1}$ and solidified with silica gel (Funk \& Krulwich, 1964). Chitinase production was assessed by production of zones of clearing on chitin agar

To show that colony radial growth rate was not limited by deficiency in constituents of the MM, strain A3(2) was also grown on medium in which the concentration of minimal nutrients of MM was doubled while the glucose concentration, agar concentration and medium depth were maintained .

Preparation and inoculation of plates. Medium depths of $2 \cdot 00,2 \cdot 75,3.25,4.25,4.75,5.75,6.75$ and $7.25 \mathrm{~mm}$ were investigated by pipetting different quantities of molten agar into Petri dishes ( $85 \mathrm{~mm}$ diameter). The depths of medium were measured by removing sections near the colonies and examining their thickness under a low power microscope. Final glucose concentrations of $0,0 \cdot 2,0 \cdot 4,0 \cdot 6,0 \cdot 8,1 \cdot 0,1 \cdot 25,2 \cdot 5$ and $5 \cdot 0 \mathrm{~g} \mathrm{l}^{-1}$ were used. Plates were solidified on a level surface and air dried. Cellophane membranes, when used, were prepared as described by Allan \& Prosser (1983). Plates were incubated in a moist environment for up to $80 \mathrm{~d}$ and showed no visible signs of dehydration at the end of this period.

Unless otherwise stated, plates were inoculated from dilutions of the stock spore suspensions prepared in $0.05 \mathrm{M}$ Tris $\mathrm{HCl}$. $\mathrm{pH} 7 \cdot 2$. Stab inoculations from suspensions containing $10^{6}$ spores $\mathrm{ml}^{-1}$ were used when investigating the effect of medium depth and glucose concentration on colony radial growth rate. A blunt mounted needle was used for inoculation of plates which had cellophane underlays. The effect of inoculum size on colony radial growth rate was tested by preparing spore suspensions of decreasing concentration from the stock suspensions of $S$. coelicolor $\mathrm{A} 3(2)$ in $0.05 \mathrm{M}$-Tris $/ \mathrm{HCl}$, pH 7.2, containing $0.01 \%$ Tween 80 . Inoculations $(5 \mu \mathrm{l})$ of each spore concentration were made centrally with a positive displacement pipette (Microman, Gilson). Unless otherwise stated all experiments were done in triplicate and plates were incubated in the dark at $30^{\circ} \mathrm{C}$ in a perspex box containing moist paper. General observations were made from these and spread plates, prepared using the same media and culture conditions as described above.

Measurement of colony grow'th. Initial colony growth was followed by photographing colonies derived from single spores growing on MM plates overlaid with cellophane membranes. Photographs were taken under a microscope (Reichert, Austria) at $2 \mathrm{~h}$ intervals after the formation of a secondary branch within the mycelial tree until diameters of approximately $400 \mu \mathrm{m}$ were achieved. Colony diameters were measured along set meridia from enlarged photographic negatives. Initially the colony margin was sparse and consequently the colony diameter was estimated as the point where the meridia were intersected by a straight line drawn between tips of hyphae on either side. Colony radial growth rate was calculated by linear regression analysis of the mean colony radius as a function of time.

In all other experiments colonies were grown for approximately $2 \mathrm{~d}$ before measurements were recorded. The increase in colony diameter was measured along the same two intersecting perpendicular lines at each time interval with a stereoscopic binocular microscope (American Optical Corporation). Measurements were initially taken at daily intervals but less frequently in older colonies. All results are quoted as mean \pm standard error (SE).

Measurement of hyphal frequency. Colonies grown on solidified $\mathrm{MM}$ of the required depth and glucose concentration and overlaid with cellophane membranes were stained for $15 \mathrm{~s}$ with $0.02 \%(\mathrm{w} / \mathrm{v})$ aqueous crystal violet solution and six photographs were taken randomly around each colony margin at $\times 80$ magnification under a phase-contrast microscope (Reichert, Austria). Negatives were projected to a known magnification and a scaling system was designed, at the same magnification, consisting of arcs $100 \mu \mathrm{m}$ long positioned $50 \mu \mathrm{m}$ apart. Hyphal frequency was arbitrarily assessed as the mean number of hyphae crossing a $100 \mu \mathrm{m}$ arc in the six areas photographed. The scaling arc was always positioned centrally at the apex of the most peripheral leading hyphae, 
i.e. the extreme colony margin. The stated hyphal frequency value was typically the mean of six colonies analysed in this manner. It should be noted in using this definition that a hypha which grew back on itself could cross the scaling arc more than once.

Variation in hyphal frequency within individual colonies was assessed from photographs of colonies of varying size with hyphal frequency analysed at 50,100,150,200,250 and $300 \mu \mathrm{m}$ behind the colony margin. These regions contained only vegetative hyphae. Aerial hyphal initials were generally apparent at approximately $350 \mu \mathrm{m}$ behind the margin. Hyphal frequency of colonies of different diameter was assessed in triplicate as the number of hyphae crossing a $100 \mu \mathrm{m}$ arc drawn $150 \mu \mathrm{m}$ and $200 \mu \mathrm{m}$ behind the colony margin for strains $\mathrm{A} 3(2)$ and $\mathrm{J} 802$ respectively. MM with final glucose concentrations of $0,0.4$ and $0.8 \mathrm{~g}^{-1}$ was used for analysis of $S$. coelicolor A3(2) and a final concentration of $0.4 \mathrm{~g} \mathrm{t}^{-1}$ for strain J802. Agar depth was maintained at $3.25 \mathrm{~mm}$. The hyphal growth unit was also calculated for colonies of strain $\mathrm{J} 802$ as a function of colony size. The total mycelial length was measured in a rectangular area $(100 \mu \mathrm{m} \times 50 \mu \mathrm{m})$ at the colony margin with a MOP 10 digital image analyser (Reichert) and a Hewlett Packard HP85 microcomputer. This length was then divided by the number of hyphal tips appearing in this area. Hyphal frequency was also measured in colonies of $1.5 \mathrm{~mm}$ radius of each strain at the medium depths and glucose concentrations given above.

\section{RESULTS}

\section{General observations}

Vegetative hyphae developed from the swollen germinated spore to form a branched mycelium which resulted in the formation of circular colonies with both strains studied. Aerial hyphal initials developed directly from the substrate hyphae approximately $35 \mathrm{~h}$ after germ tube outgrowth. The aerial hyphae were wider and less branched and the majority differentiated into spore chains. Lysis of vegetative mycelium supporting aerial hyphae was observed. Germinating spores were occasionally observed close to the margin of mature colonies in heavily inoculated plates where vegetative mycelium was either sparse or lysed. This phenomenon has previously been reported in S. coelicolor (Hopwood, 1960; Wildermuth, 1970) but was only evident in the initial spores differentiated from a developing colony. Wildermuth (1970) proposed that the relative humidity close to the growth medium was possibly high enough to initiate germination and that lysis of vegetative mycelia was crucial to the development of aerial mycelia. We only observed germination in situ when plates were crowded, under which conditions lysis of vegetative mycelia could provide the nutrients required for spore germination and further mycelial development. This is consistent with the observation of renewed growth of both substrate and aerial mycelia from the centre of large colonies. It has been suggested that the low relative humidity in growing colonies will normally prevent this in situ germination. The hyphal clusters which result from each spore in a chain producing a germ tube (usually only one germ tube was observed protruding from each spore under these conditions) indicate a much more regular branch pattern than that of an unrestricted colony margin.

Plates inoculated centrally developed a single colony which grew to cover the whole Petri dish. In spread plates discrete colonies were formed whose final diameter decreased as the concentration of the spore inoculum increased. In crowded plates mycelium at the colony margin was more sparse and less branched while growth of approaching colonies slowed down and was often associated with production of a blue pigment (Rudd \& Hopwood, 1979). Occasionally, however, unrestricted margins merged to continue growth as a single colony with sporulation and pigmentation bands coinciding. Mature colonies exhibited sectors similar to those described for colonies of filamentous fungi (Pontecorvo \& Gemmell, 1944). Some sectors were observed that consisted of either substrate hyphae alone or both substrate and aerial hyphae, while others consisted of regions of pigment production. These different morphologies were also observed as banding patterns, which occasionally extended to the whole colony. Growth patterns were very variable, particularly with regard to pigmentation, with bands changing colour during colony development, presumably due to local $\mathrm{pH}$ changes. When inoculated directly onto agar, hyphae grew within the medium. When this was prevented by inoculation onto a cellophane membrane the mycelial mat was more dense, colonies were densely pigmented and formation of aerial mycelium increased. 
Agar supported continued growth of the wild-type strain but strain J802 was unable to metabolize the products of agar breakdown and growth on purified agar was very poor and eventually ceased. Diffusion of agarase through cellophane underlays was limited but some agar degradation did occur below the centre of old colonies. Chitinase was also produced by both strains.

\section{Effects of light, minimal nutrient concentration and inoculum concentration}

Colony radial growth rates of $S$. coelicolor A3(2) grown under constant illumination and in the dark were $28.24 \mu \mathrm{m} \mathrm{h}^{-1}(\mathrm{SE} 0.75, n=6)$ and $28.64 \mu \mathrm{m} \mathrm{h}^{-1}(\mathrm{SE} 2.32, n=6)$ respectively. These values are not significantly different at the $5 \%$ level of significance. $S$. coelicolor A3(2) was grown on $0.4 \mathrm{~g}$ glucose $1^{-1}$ at a medium depth of $3.25 \mathrm{~mm}$ with single and double strength minimal nutrient concentrations, giving colony radial growth rates during the primary phase of growth (see below) of $22.4 \mu \mathrm{m} \mathrm{h}^{-1}($ SE $0.34, n=6)$ and $21.58 \mu \mathrm{m} \mathrm{h}^{-1}($ SE $0.62, n=6)$ respectively. These values are not significantly different ( $5 \%$ level of significance), showing that minimal nutrients did not limit radial extension. However, the increased minimal nutrient concentration significantly decreased production of blue diffusible pigment and prolonged the primary linear phase of growth. Analysis of variance showed that colony radial growth rate was independent of inoculum spore concentration, with a rate of $14.23 \mu \mathrm{m} \mathrm{h}^{-1}$ (SE $0 \cdot 30, n=7$ ) for values of spore concentration in the range of $10^{4}-10^{7}$ spores $\mathrm{ml}^{-1}$, and in subsequent experiments stab or surface inocula were made from diluted stock spore suspensions of approximately $10^{6}$ spores $\mathrm{ml}^{-1}$.

\section{Initial colony radial growth rate}

Colony radial growth observed microscopically was initially exponential $\left(0.067 \mathrm{~h}^{-1}\right)$, despite linear extension of individual hyphae. This rate was much less than the specific growth rate (Allan \& Prosser, 1983) and may have been due to the lack of a well defined margin, often through growth of hyphae towards the colony centre, and consequent difficulties in measuring the diameter. In older colonies marginal hyphal frequency was greater and the diameter could be measured accurately. Colony growth could then be described as a series of two or more phases of linear increases in diameter, at successively decreasing rates. The first two phases are termed primary and secondary and linear rates of growth calculated during these periods are termed primary and secondary radial growth rates respectively.

\section{Effect of medium depth on colony radial growth rate}

The primary and secondary colony radial growth rates for $S$. coelicolor A3(2) were independent of medium depth, with values of $14.26 \mu \mathrm{m} \mathrm{h}^{-1}(\mathrm{SE} 0.45, n=8)$ and $21.03 \mu \mathrm{m} \mathrm{h}^{-1}$ (SE $0.45, n=8$ ) respectively for medium depths between 2.00 and $7.25 \mathrm{~mm}$, and the number of phases observed was greatest at low medium depths. At depths greater than $4.75 \mathrm{~mm}$ radial growth was biphasic, with an additional gradual decrease occurring as the colony margin approached the edge of the Petri dish. The duration of the primary phase increased with medium depth up to $4.5 \mathrm{~mm}$ (Fig. 1). Colonies of strain J802 only exhibited biphasic growth up to depths of $4.75 \mathrm{~mm}$ (Fig. 2). The duration of the primary phase was relatively short and variable (140-400 h), showing no correlation with medium depth. The fact that primary colony radial growth rate of strain $\mathrm{J} 802$ was slower than the secondary phase (a characteristic never observed in the wild-type) and also that colony radial growth rate increased shortly after agarase production was observed, indicate that this strain can utilize the products of agar degradation, but only to a limited extent. This may be evident only when colony radial growth rate is limited by other factors (e.g. medium depth). The continued formation of a sparse mycelial mat indicated that the mutant had not reverted. The secondary colony radial growth rate was independent of medium depth (Fig. 2). When grown on cellophane membranes, colony growth was not multiphasic and diameter increased at a constant, linear rate of $23.93 \mu \mathrm{m} \mathrm{h}^{-1}$ (SE 0.69 , $n=8)$ which was independent of medium depth between 2.00 and $7.25 \mathrm{~mm}(5 \%$ level of significance). 


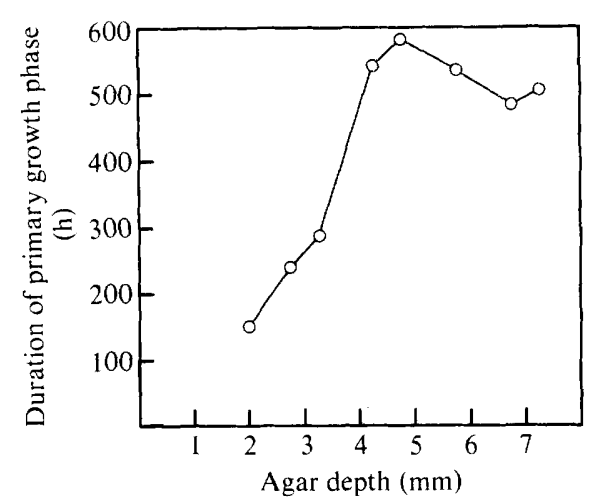

Fig. 1. Influence of medium depth on the duration of the primary growth phase of $S$. coelicolor A3(2).

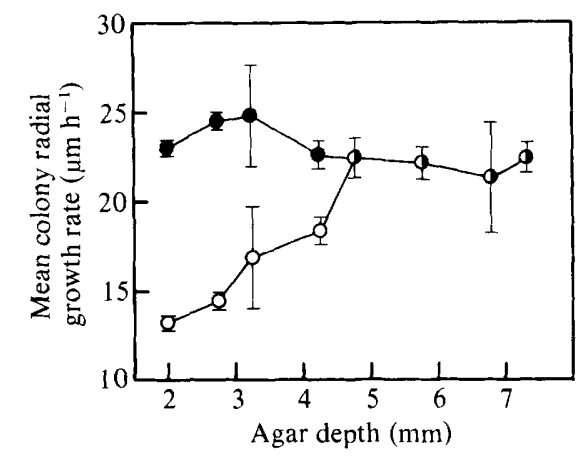

Fig. 2

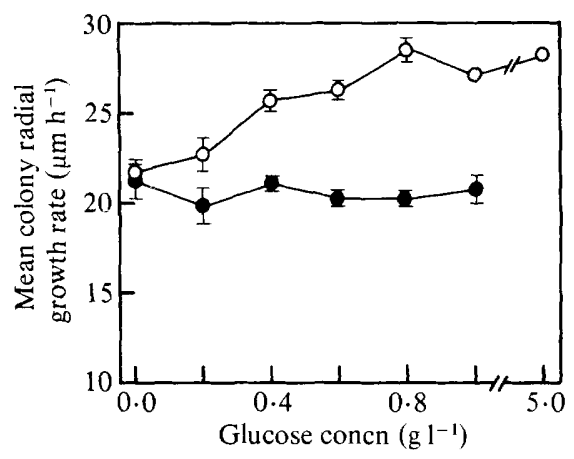

Fig. 3

Fig. 2. Influence of medium depth on the primary $(O)$ and secondary $(O)$ colony radial growth rates of $S$. coelicolor $\mathbf{J} 802$ in the absence of cellophane. Bars represent SE.

Fig. 3. Influence of glucose concentration on the primary and secondary colony radial growth rates of $S$. coelicolor $\mathrm{J} 802$ with $(\mathrm{O})$ and without (O) cellophane. Bars represent SE.

\section{Effect of glucose concentration on colony radial growth rate}

Medium containing $1 \mathrm{mg}$ glucose $1^{-1}$ and solidified with silica gel supported growth and sporulation of both strains of $S$. coelicolor. In the experiments described below medium was solidified using Oxoid Agar No. 3. Impurities in the agar allowed growth of both strains without added glucose, although the mycelial mat of $\mathrm{J} 802$ was more sparse than that of the wild-type. A glucose concentration of $1 \mathrm{~g} \mathrm{l}^{-1}$ inhibited growth and development of the wild-type. Growth occurred for less than $100 \mathrm{~h}$, hyphae were highly branched and sporulation and pigmentation were inhibited. Primary colony radial growth rate of strain A3(2) was $22.98 \mu \mathrm{m} \mathrm{h}^{-1}$ (SE $0.33, n=$ 6) at glucose concentrations between 0.0 and $1.0 \mathrm{~g}^{-1}$. Both this rate and the primary colony radial growth rate of strain $\mathrm{J} 802$ (Fig. 3) grown without cellophane were independent of glucose concentration ( $1 \%$ level of significance), although the mycelial mat appeared visually to be thicker and the colony height to be greater at high glucose concentrations. When grown on cellophane membranes, primary colony radial growth rate of strain J802 was generally greater and also increased with glucose concentration up to $0.6 \mathrm{~g} \mathrm{l}^{-1}$ (Fig. 3). Increased colony radial growth rate was also observed for strain $\mathrm{A} 3(2)$ when grown on a cellophane membrane with a mean initial value of $27.6 \mu \mathrm{m} \mathrm{h}^{-1}$ (SE 1.0,n=5) compared to $23.9 \mu \mathrm{m} \mathrm{h}^{-1}(\mathrm{SE} 0.03, n=5)$ in the absence of cellophane. 

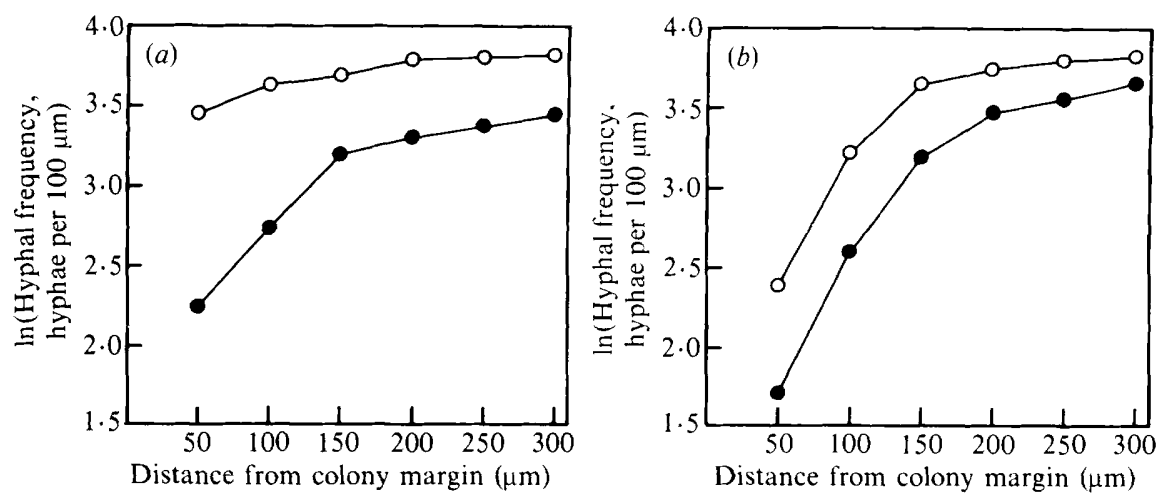

Fig. 4. Variation in hyphal frequency of $S$. coelicolor A3(2) (a) and J802 (b) with distance from the margin of colonies of radius $1.5 \mathrm{~mm}(\mathrm{O})$ and $4.5 \mathrm{~mm}(\mathcal{O})$. Hyphal frequency was assessed as the number of hyphae crossing a $100 \mu \mathrm{m}$ arc placed at varying distances behind the colony margin.

\section{Variation in hyphal frequency within individual colonies}

Hyphal frequency increased exponentially with distance from the colony margin in both strains (Figs $4 a$ and $4 b$ ) and approached constant values at $200 \mu \mathrm{m}$ from the margin. Hyphal frequency was always higher at the margins of smaller colonies. Although maxima were similar for both strains, hyphal frequency of strain $\mathrm{J} 802$ decreased more sharply towards the margin and was significantly lower than that of the wild-type at $50 \mu \mathrm{m}$. In young colonies of strain A3(2) the maximum was almost reached at $50 \mu \mathrm{m}$. This is in accordance with visual observations of mycelial mats of both strains.

\section{Variation in hyphal frequency during colony growth}

Hyphal frequency at the colony margin was plotted as a function of colony size for strains A3(2) and J802 (Figs 5 and 6, respectively). Hyphal frequency of strain A3(2) varied in a similar manner at all three glucose concentrations tested. In colonies of radius less than $2 \mathrm{~mm}$ the hyphal frequency was high (approximately 40 hyphae per $100 \mu \mathrm{m}$ ) but as colony radius increased further hyphal frequency decreased, with values ranging from 20 to 27 hyphae per $100 \mu \mathrm{m}$ ). However, neither the rate of decrease nor the colony size at which the new constant hyphal

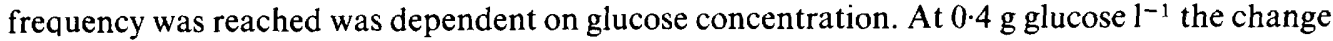
from high (approximately 40 hyphae per $100 \mu \mathrm{m}$ ) to low (approximately 23 hyphae per $100 \mu \mathrm{m}$ ) levels of hyphal frequency occurred abruptly, whereas at 0 and $0.8 \mathrm{~g}$ glucose $1^{-1}$ the variation was more gradual. Visual observations suggested that the low level of hyphal frequency attained in these colonies remained relatively constant as the colony radius increased further but it was not possible to test this in colonies of radius greater than $7 \mathrm{~mm}$ because of aggregation of the peripheral hyphae following staining. The hyphal frequency of the non-agar-utilizing mutant, grown at $0.4 \mathrm{~g}$ glucose $1^{-1}$ (Fig. 6), was initially higher than that of the wild-type, but gradually declined (from 44 to 39 hyphae per $100 \mu \mathrm{m}$ ) before abruptly decreasing to a relatively constant value of 28 hyphae per $100 \mu \mathrm{m}$.

Generally, as colonies increased in radius, both the number of hyphal tips and the total mycelial length per unit area decreased. However, these parameters did not decrease proportionately and consequently the hyphal growth unit increased. The mean hyphal growth unit of colonies of strain J802 increased from $33.18 \mu \mathrm{m}$ (SE 1.29) at colony radii less than $3 \mathrm{~mm}$ to $41.11 \mu \mathrm{m}$ (SE 2.42) in colonies of radius greater than $3 \mathrm{~mm}$, indicating a significant increase in branching initiation in the latter (Table 1). The former value is similar to the mean hyphal growth unit of 12 young colonies of $S$. coelicolor A3(2) (Allan \& Prosser, 1983).

\section{Effect of medium depth and glucose concentration on hyphal frequency}

The hyphal frequency of $S$. coelicolor A3(2) exhibited some dependency on medium depth up to approximately $5 \mathrm{~mm}$ with frequency remaining relatively constant at greater depths (Fig. 7). 


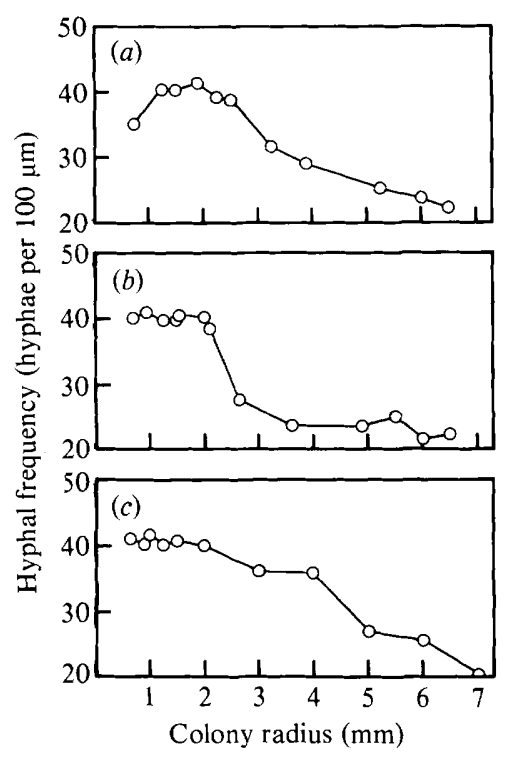

Fig. 5

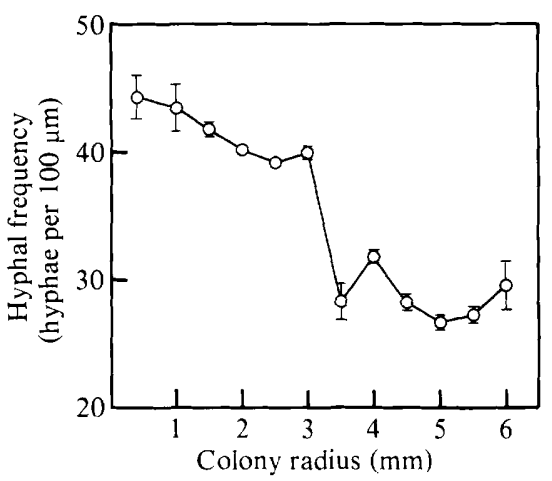

Fig. 6

Fig. 5. Variation in hyphal frequency of $S$. coelicolor A3(2) with colony size on MM with glucose concentrations $\left(\mathrm{g} \mathrm{l}^{-1}\right)$ of $0(a), 0.4(b)$ and $0.8(c)$. Hyphal frequency was assessed as the number of hyphae crossing a $100 \mu \mathrm{m}$ arc placed $150 \mu \mathrm{m}$ behind the colony margin.

Fig. 6. Variation in hyphal frequency of $S$. coelicolor J802 with colony size on MM with a glucose concentration of $0.4 \mathrm{~g} \mathrm{I}^{-1}$. Hyphal frequency was assessed as the number of hyphae crossing a $100 \mu \mathrm{m}$ arc placed $200 \mu \mathrm{m}$ behind the colony margin. Bars represent SE.

Table 1. Variation in total mycelial length, number of tips and hyphal growth unit in a defined area with increasing colony radius of S. coelicolor J802

Each value is the mean of three measurements except for those marked *, where four colonies were measured. The overall mean hyphal growth unit was calculated for colonies of radius less than and greater than $3 \mathrm{~mm}$, the size at which hyphal frequency changed significantly. Standard errors are given for the hyphal growth unit.

$\begin{array}{cccc}\begin{array}{c}\text { Colony } \\ \text { radius } \\ (\mathrm{mm})\end{array} & \begin{array}{c}\text { Mean total } \\ \text { mycelial length } \\ (\mu \mathrm{m})\end{array} & \begin{array}{c}\text { Mean no. of } \\ \text { hyphal tips }\end{array} & \begin{array}{c}\text { Mean hyphal } \\ \text { growth unit } \\ (\mu \mathrm{m})\end{array} \\ 0.4 & 942.8 & 29.33 & 32.63 \pm 1.97 \\ 1.0 & 724.8 & 24.33 & 29.80 \pm 1.47 \\ 1.5 & 895.6 & 26.33 & 34.17 \pm 3.16 \\ 2.0 & 819.3 & 26.67 & 30.49 \pm 3.81 \\ 2.5^{*} & 557.2 & 15.00 & 38.64 \pm 3.57 \\ 3.0^{*} & 679.6 & 21.00 & 33.35 \pm 2.91 \\ 3.5^{*} & 569.9 & 12.50 & 46.53 \pm 2.35 \\ 4.0^{*} & 542.7 & 16.25 & 34.93 \pm 4.23 \\ 5.0 & 436.7 & 12.33 & 35.81 \pm 3.44 \\ 6.0 & 512.5 & 11.33 & 45.39 \pm 1.15 \\ 7.0 & 371.9 & 8.66 & 42.90 \pm 0.33\end{array}$

\section{Overall mean hyphal growth unit $(\mu \mathrm{m}$ per tip)}

33.18 (SE 1.29)

$41 \cdot 11$ (SE 2.42)

This dependency was much greater for strain $\mathrm{J} 802$ which showed a two-fold increase in frequency over the range of depths investigated. The hyphal frequency of colonies of strain A3(2) was independent of glucose concentration (Fig. 8), but increased in colonies of strain J802 up to a concentration of $1.0 \mathrm{~g} \mathrm{t}^{-1}$. This was not the case for strain $\mathrm{J} 802$ growing on a cellophane membrane and hyphal frequency of this strain showed no further increase at high glucose concentrations. 


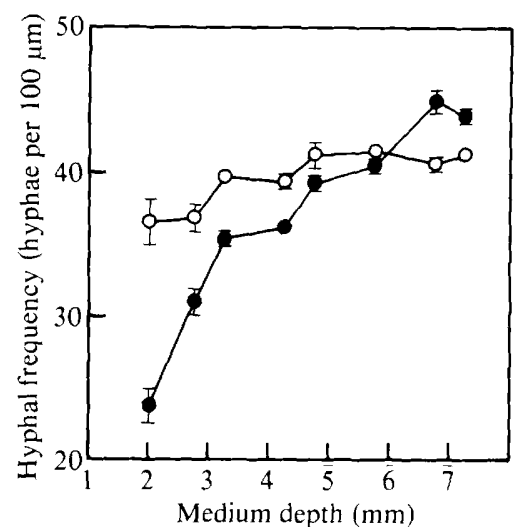

Fig. 7

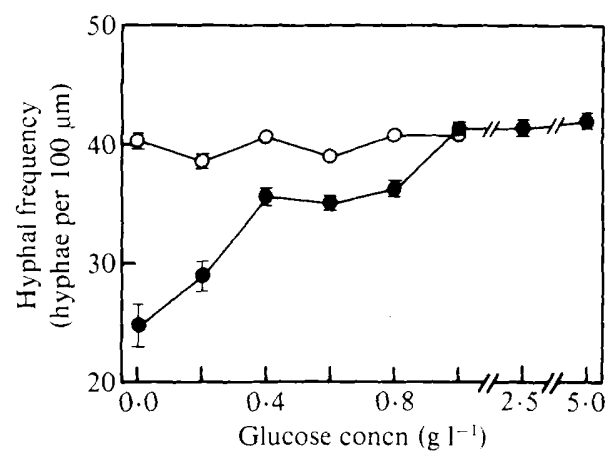

Fig. 8

Fig. 7. Variation in hyphal frequency of $S$. coelicolor A3(2) (O) and J802 (O) with medium depth Hyphal frequency was assessed as the number of hyphae crossing a $100 \mu \mathrm{m}$ arc placed $150 \mu \mathrm{m}$ behind the colony margin. Bars represent SE.

Fig. 8. Variation in hyphal frequency of $S$. coelicolor $\mathrm{A} 3(2)(\mathrm{O})$ and $\mathrm{J} 802(\mathrm{O})$ with glucose concentration. Hyphal frequency was assessed as the number of hyphae crossing a $100 \mu \mathrm{m}$ arc placed $150 \mu \mathrm{m}$ behind the colony margin. Bars represent SE.

\section{DISCUSSION}

The filamentous growth habit of $S$. coelicolor is believed to confer similar advantages for growth on solid media to those proposed for filamentous fungi. Thus, it permits hyphal growth beyond the region of nutrient depletion with further colonization behind the margin. A single colony may therefore grow to cover a Petri dish, while colonies of unicellular organisms produce self-limiting, discrete colonies. As in filamentous fungi, regulation of biomass production also permits growth at very low nutrient levels $\left(1 \mathrm{mg}^{-1}\right)$ which are lower than the minimum threshold concentration required for growth of unicellular prokaryotes such as Escherichia coli (90 $\left.\mathrm{mg} \mathrm{1}^{-1}\right)$, Klebsiella aerogenes $\left(13 \mathrm{mg} \mathrm{1}^{-1}\right)$ and Streptococcus faecalis (Pirt, 1967). Substrate utilization is further increased by growth into the agar which is presumably assisted in $S$. coelicolor by production of a diffusible agarase. This is evident in that the submerged hyphae are frequently observed in advance of the growing surface colony margin.

Difficulties in measuring initial, microscopically observed increases in colony diameter indicated less tight regulation of mycelial growth and branching than in filamentous fungi, although Plomley (1959) had similar problems with Chaetomium globosum. Subsequent colony extension is linear and conforms with the hypothesis of Pirt (1967) for bacterial colony growth and its extension to fungal growth (Trinci, 1971). This is consistent with the existence of an outer peripheral ring of hyphae, the peripheral growth zone, within which biomass production is exponential while individual hyphae, and hence the colony margin, extend at a constant rate. Colony radial growth rates varied with environmental conditions but were equivalent to the range of hyphal extension rates in mature colonies (Allan \& Prosser, 1983).

The effects of medium depth and glucose concentration on the primary phase of colony radial growth rate are summarized in Table 2 . In unicellular bacteria, colony radial growth typically increases with both substrate concentration and agar depth. This is due to effective extension of the zone from which nutrient may be depleted, in the first case by increasing the concentration of substrate entering the zone and in the second by increasing the time before diffusion from below ceases. Colony radial growth rate in fungi is typically independent of medium depth. This is due to a reduction in branching at low medium depths, such that nutrient at the margin does not become limiting, and it is also believed that marginal hyphae extend at such a rate as to escape the nutrient depletion zone. The influence of glucose concentration is less well defined. Trinci (1969) observed an increase in colony radial growth rate with glucose concentration for 
Table 2. Summary of the effects of medium depth and glucose concentration on the primary colony radial growth rate of $S$. coelicolor $A 3(2)$ and $J 802$

Primary colony radial growth rate as a function of:

Strain A3(2) Strain J802

Medium depth

With a cellophane underlay

Without a cellophane underlay

Glucose concentration

With a cellophane underlay

Without a cellophane underlay

$\begin{array}{cc}\text { ND } & \begin{array}{c}\text { Independent } \\ \text { Dependent }\end{array} \\ \text { Independent } & \begin{array}{c}\text { Dependent } \\ \text { Independent }\end{array}\end{array}$

ND, Not determined.

Aspergillus nidulans and Mucor hiemalis, up to 200 and $75 \mathrm{mg} \mathrm{1}^{-1}$, beyond which there was a decrease. Colony radial growth rate of Penicillium chrysogenum was independent of glucose concentration in the range $0 \cdot 01-1.0 \mathrm{~g}^{-1}$.

Primary colony radial growth rate of $S$. coelicolor A3(2), as in fungi, is independent of medium depth while hyphal frequency decreases at medium depths below $4.75 \mathrm{~mm}$. This suggests that overall growth is limited at these small depths by resiriction of diffusion of nutrients from below the colony, while colony expansion is maintained by a reduction in hyphal frequency. The lack of dependence of both primary colony radial growth rate and hyphal frequency on glucose concentration is thought to be due to utilization of agar, which acts as an additional carbon and energy source. However, strain A3(2) is inhibited by glucose concentrations greater than $1 \mathrm{~g} \mathrm{l}^{-1}$, which is higher than the concentrations required to inhibit $A$. nidulans and $M$. hiemalis. The effect of such high concentrations is different, causing cessation of growth rather than a reduction in colony radial growth rate. Glucose toxicity effects have been observed in unicellular bacteria (Pirt, 1967) and filamentous fungi (Panikov et al., 1981).

Strain J802 does not metabolize agar and in the absence of a cellophane underlay its colony radial growth rate increased with medium depth but was independent of glucose concentration. The former effect may be explained by its inability to utilize agar and consequently its growth will be restricted by nutrient depletion at low medium depths. However, colony thickness, assessed visually, increased with increasing glucose concentration, indicating redistribution of increased biosynthesis to branches, rather than to tip extension. The reverse situation was found when strain J802 was grown on a cellophane underlay, where colony radial growth rate was independent of depth and proportional to glucose concentration. Hyphal frequency again increased with medium depth up to $4.75 \mathrm{~mm}$ and also increased with glucose concentration.

The lack of inhibition at $5 \mathrm{~g}$ glucose $\mathrm{I}^{-1}$ may be because the cellophane membrane prevented growth into the medium such that growth became dependent on diffusion of nutrients through the membrane. This may also explain the effects of glucose concentration on strain J802 grown with and without a cellophane membrane. An alternative explanation is that, during growth on cellophane, secondary metabolites may be localized within the colony, rather than diffusing through the agar to the colony margin. This will again reduce toxicity effects, giving the increase in colony radial growth with increasing glucose observed during growth on cellophane.

Hyphal frequency changes within colonies of $S$. coelicolor are similar to those of the filamentous fungi in that hyphal frequency increases exponentially from the colony margin towards the centre and attains a constant value before formation of aerial hyphae. This results from exponential branch formation in the peripheral growth zone, with continued exponential growth being prevented by either nutrient limitation, inhibition by secondary metabolites or interhyphal interference. The constant saturation frequency achieved in both strains of $S$. coelicolor was significantly lower in older colonies (radii greater than $4.5 \mathrm{~mm}$ ) and these lower branch frequencies remained constant at distances greater than $200 \mu \mathrm{m}$ behind the colony margin and were thought to be maintained without further fluctuation. Similar characteristics have been observed in colonies of Chaetomium globosum grown on water agar compared to those grown on malt agar (Plomley, 1959). 
This suggests a response by marginal mycelia to changing conditions as the colony ages. This was confirmed for both strains by an arbitrary measure of hyphal frequency and, for strain J802, by estimating the value of the hyphal growth unit. The latter increased from $33.2 \mu \mathrm{m}$ (at radii less than $3 \mathrm{~mm}$ ) to $41.1 \mu \mathrm{m}$ (at radii greater than $3 \mathrm{~mm}$ ) while at these same colony sizes the mean hyphal frequency decreased from 41.5 to 28.1 hyphae per $100 \mu \mathrm{m}$. Since the initial hyphal frequency is constant during the primary growth phase, the specific rate of branch initiation must decline. In all cases, hyphal frequency decreased quite sharply from relatively constant values to lower constant values. This decrease is thought to represent control of branching which allows a mycelium to maintain its exploitation of a surface at the expense of branch initiation, should nutrient be depleted at the colony margin, or alternatively it may be caused by secondary metabolite production. Steele \& Trinci (1975) observed differences in extension rate and hyphal diameter in hyphae of young colonies of Neurospora crassa and those at the margin of mature colonies, terming the two types of mycelia undifferentiated and differentiated. K. M. McLean \& J. I. Prosser (unpublished results) investigated changes in hyphal diameter, extension rates and branch angles in $N$. crassa during colony development and found all three properties to change sharply from constant values in young colonies to new constant values in mature colonies. They attributed such changes to the effect of secondary metabolites or staling products, produced at the colony centre at a stage during colony development, which then diffused to the margin. Although hyphal diameter, extension rate and branch angle were not measured in $S$. coelicolor, the observed changes in hyphal frequency suggest similar effects.

It is proposed that multiphasic growth of the wild-type also results from inhibition of hyphal extension by secondary metabolite production. Increasing minimal nutrient concentration decreased levels of pigmentation, and presumably secondary metabolite production in general by altering nitrogen and/or inorganic phosphate concentrations. The linear increase in radius of colonies grown at normal $\mathrm{MM}$ concentrations began to fall off at $55 \mathrm{~mm}$ diameter compared to $65 \mathrm{~mm}$ in colonies grown on double strength $\mathrm{MM}$, almost all the available surface area being colonized on these latter plates. In addition, the number of phases of growth of the wild-type strain increased and the duration of the primary phase decreased at shallow depths, where secondary metabolites will accumulate at a faster rate due to restriction of volume. In strain J802 there was only one phase of linear growth at greater medium depths. It therefore appears that for $S$. coelicolor A3(2) the accumulation of secondary metabolites and staling compounds is of equal importance to nutrient limitation in colony growth and development.

E. J. A. acknowledges receipt of a NERC Research Studentship.

\section{REFERENCES}

Allan, E. J. \& Prosser, J. I. (1983). Mycelial growth and branching of Streptomyces coelicolor A3(2) on solid medium. Journal of General Microbiology 129 , 2029-2036.

BoNNER, J. T. (1973). Development in lower organisms. Symposia of the Society for General Microbiology 23, $1-7$.

Bradley, S. G. (1978). Physiology of actinomycetes. Actinomycetes 13, 102-121.

COOPER, A. L., Dean, A. C. R. \& Hinshelwood, C. (1968). Factors affecting the growth of bacterial colonies on agar plates. Proceedings of the Royal Society B171, 173-199.

Funk, H. B. \& KRULwiCh, T. A. (1964). Preparation of clear silica gels that can be streaked. Journal of Bacteriology 88, 1200-1201.

Hodgson, D. A. \& Chater, K. F. (1981). A chromosomal locus controlling extracellular agarase production by Streptomyces coelicolor A3(2), and its inactivation by chromosomal integration of plasmid SCP1. Journal of General Microbiology 124, 339-348.
Hopwood, D. A. (1960). Phase-contrast observations on Streptomyces coelicolor. Journal of General Microbiology 22, 295-302.

Palumbo, S. A., Johnson, M. G., Rieck, V. T. \& WiTTER, L. D. (1971). Growth measurements on surface colonies of bacteria. Journal of General Microbiology 66, 137-143.

Panikov, N. S., Afremova, V. D. \& Aseeva, I.V. (1981). Kinetics of growth of colonies of the fungi Mucor plumbeus and Mortierella ramanniana on agarised media with glucose. Mikrobiologiya 50, 3843 (in English).

PARK, D. \& Robinson, P. M. (1966). Aspects of hyphal morphogenesis in fungi. In Trends in Plant Morphogenesis, pp. 27-44. Edited by E. G. Cutter. London: Longmans.

PIRT, S. J. (1967). A kinetic study of the mode of growth of surface colonies of bacteria and fungi. Journal of General Microbiology 47, 181-197.

PIRT, S. J. (1975). Growth of microbial colonies on the surface of solid medium. In Principles of Microbe and 
Cell Cultivation. Oxford \& London; Blackwell Scientific Publications.

Plomley, N. J. B. (1959). Formation of the colony in the fungus Chaetomium. Australian Journal of Biological Sciences 12, 53-64.

Pontecorvo, G. \& Gemmell, A. R. (1944). Colonies of Pencillium notatum and other moulds as models of the study of population genetics. Nature, London 154, 532-534.

Pridham, T. G. \& Tresner, H. D. (1974). Family VII; Streptomycetaceae. Waksman and Henrici (1943). In Bergey's Manual of Determinative Bacteriology, 8th edn. Edited by R. E. Buchanan \& N. E. Gibbons. Baltimore: Williams \& Wilkins.

Reyrolle, J. \& Letellier, F. (1979). Autoradiographic study of the localization and evolution of growth zones in bacterial colonies. Journal of General Microbiology 111, 399-406.

RiesenberG, D. \& Bergter, F. (1979). Dependence of macromolecular composition and morphology of Streptomyces hygroscopicus on specific growth rate. Zeitschrift für allgemeine Mikrobiologie 19, 415-430.

Robinson, P. M. \& Smith, J. M. (1979). Development of cells and hyphae of Geotrichum candidum in chemostat and batch culture. Transactions of the British Mycological Society 72, 39-47.

Rudd, B. A. M. \& Hopwood, D. A. (1979). Genetics of actinorhodin biosynthesis by Streptomyces coelicolor A3(2). Journal of General Microbiology 114, 35-43.

Shirling, E. B. \& Gottlieb, D. (1968). Cooperative description of type cultures of Streptomyces. II. Species descriptions from first study. International Journal of Systematic Bacteriology 18, 69-189.

Steele, G. C. \& Trinci, A. P. J. (1975). Morphology and growth kinetics of hyphae of differentiated and undifferentiated mycelia of Neurospora crassa. Journal of General Microbiology 91, 362-368.

TRINCI, A. P. J. (1969). A kinetic study of the growth of Aspergillus nidulans and other fungi. Journal of General Microbiology 57, 11-24.

TrinCI, A. P. J. (1971). Influence of the width of the peripheral growth zone on the radial growth rate of fungal colonies on solid media. Journal of General Microbiology 67, 325-344.

TrincI, A. P. J. (1974). A study of the kinetics of hyphal extension and branch initiation of fungal mycelia. Journal of General Microbiology 81, 225236.

Wimpenny, J. W. T. \& Lewis, M. W. A. (1977). The growth and respiration of bacterial colonies. Journal of General Microbiology 103, 9-18.

WILDERMUTH, H. (1970). Development and organization of the aerial mycelium in Streptomyces coelicolor. Journal of General Microbiology 60, 43-50. 